\title{
Insights and treatment options for psychiatric disorders guided by functional MRI
}

\author{
Tonmoy Sharma \\ Clinical Neuroscience Research Centre, Dartford, Kent, United Kingdom \\ J. Clin. Invest. 112:10-18 (2003). doi:10.1172/JCI200319166.
}

\section{Introduction}

The advent of functional imaging has led to a new wave of insights into the pathophysiology and treatment of psychiatric disorders. In this Perspective, schizophrenia is used as an example of such a psychiatric disorder to illustrate these advances. Schizophrenia is a chronic and debilitating disorder characterized by a heterogeneous group of symptoms, including positive symptoms (delusions, hallucinations, disorganized thought), negative symptoms (flattened affect, stereotyped thinking, difficulty in abstract thinking), and multifaceted cognitive deficits, most prominently in the areas of attention, memory, and executive functioning (1). Emil Kraepelin gave us the first clinical account of schizophrenia in 1896 . He named it dementia praecox, literally, early or premature deterioration of one's mental faculties. In using this term, Kraepelin was referring to the fact that the disorder often strikes in adolescence or early adulthood and runs a chronic, lifelong, disabling course. In his writing about the disorder, Kraepelin emphasized the evident cognitive impairment in individuals that he studied, which comprised deficits in attention, motivation, problem solving, learning, and memory. He was also the first to link these cognitive impairments with poor functional outcome in patients with schizophrenia, such as deficits in social functioning, independent living, and self-care abilities. The term "schizophrenia" (schizo, split; phreno, mind) was coined by the Swiss psychiatrist Eugen Bleuler in 1911. Although Bleuler disputed Kraepelin's assumption that the disorder struck only in early adulthood and

\footnotetext{
Address correspondence to: Tonmoy Sharma, Clinical Neuroscience Research Center, 7 Twisleton Court, Priory Hill, Dartford, Kent DA2 6AU, United Kingdom. Phone: 44-1322-286863; Fax: 44-1322-286861;

E-mail: t.sharma@psychmed.org.uk.

Conflict of interest: The author has declared that no conflict of interest exists.

Nonstandard abbreviations used: functional MRI (fMRI); prefrontal cortex (PFC); dorsolateral prefrontal cortex (DLPFC); mental state attribution (MSA); blood oxygen level-dependent (BOLD); positron emission tomography (PET); prepulse inhibition (PPI); obsessive-compulsive disorder (OCD); procedural learning $(\mathrm{PL})$.
}

always entailed dementia-like cognitive degeneration, he agreed that cognitive impairment was a core feature of schizophrenia, or its fundamental symptom. Other "peculiar" features, such as hallucination and delusions, were considered by Blueler to be secondary or "accessory" symptoms.

The discovery of antipsychotics in the 1950s revolutionized the treatment of schizophrenia and directed emphasis toward positive symptoms. At that time, treatment with antipsychotics, with their encouraging effect on psychotic symptoms, led to the closure of most hospitals for chronic patients with severe and enduring mental illness and offered much hope for these patients to be reintegrated into society. However, by the 1960s it became obvious that a reduction in positive symptoms did not lead to recovery from schizophrenia and did not significantly improve functional outcome (2). The understanding of the fundamental deficits in schizophrenia came full circle as it came to be accepted that cognitive dysfunction plays a central role in the illness, just as Kraepelin and Bleuler had suggested at the dawn of the 20th century. Cognitive deficits are a core feature of schizophrenia, which (a) may precipitate psychotic and negative symptoms (3); (b) are relatively stable over time, with progressive deterioration after the age of 65 in some patients (4); (c) persist upon the remission of psychotic symptoms (5); (d) are related to but separate from negative symptoms $(6,7)$; and (e) determine functional impairment characteristic of the patients with this disorder (8).

Functional brain imaging in schizophrenia was originally intended to elucidate the underlying physiological disturbances that lead to manifest illness. This Perspective will deal mainly with attempts by researchers to understand the pathophysiology and the mechanism of drug action using functional magnetic resonance imaging ( $\mathrm{MMR})$, a technique that has the obvious advantages of minimal invasiveness, no radioactivity, widespread availability, and virtually unlimited study repetitions. These attributes make it ideally suited to the study of in vivo brain function in psychiatric illness like schizophrenia, facilitating the characterization of developmental changes in brain function from childhood and throughout the clinical course of the illness, and in 
response to treatment. In this article, some current issues surrounding the interpretation of functional imaging data in schizophrenia will be considered. Various investigators have examined links between brain activation, cognitive functioning, and symptoms, in the hope of identifying those regions and circuits that may be abnormal in schizophrenia. Ultimately, fMRI may help delineate disease pathways based upon pathophysiological correlates that may complement nosology. If this is successful, neural network models might be built to emulate brain malfunctions for each disease. This, in turn, may lead to better therapeutic options.

\section{Defining the deficits}

The realization that functional outcome in schizophrenia is related mainly to cognitive deficits and less to psychotic symptoms (8) has led to the exploration of various cognitive-enhancing techniques for the treatment of this disorder. These have encompassed both pharmacological and psychological methods. To understand the pathophysiology of schizophrenia and to eventually develop specific treatments for cognitive remediation and disability reduction in schizophrenia, we need to understand the functional brain circuitry associated with impaired cognition.

In 1919, Kraepelin noted the similarities between the negative or deficit symptoms of schizophrenic patients and those of patients with frontal lobe lesions (9). Postmortem neuropathology has found prefrontal cortex (PFC) abnormalities, including reduced neuropil (intraneuronal volume) without neuronal loss in dorsal PFC. This reduction was linked to Brodmann areas 9 and 46 (Brodmann areas are regions of the cerebral cortex mapped on the basis of the cortical cytoarchitectural patterns) $(10,11)$. Other PFC abnormalities exposed by postmortem neuropathology include diminished inhibitory inputs from prefrontal chandelier cells onto the axonal processes of dorsal PFC pyramidal neurons (12), and reductions in the abundance and metabolic activity of dorsal PFC interneurons $(13,14)$.

Early fMRI studies involving patients with schizophrenia noted a reversal of the antero-posterior gradient of cerebral blood flow in healthy controls (15), a phenomenon termed hypofrontality (16). However, a comparable number of studies have failed to find evidence of hypofrontality or have instead observed a hyperfrontal response in schizophrenic patients (17). The use of the term hypofrontality has also been extended to describe a failure of task-induced frontal cerebral response. Indeed, this has proved to be a reliable finding. Numerous studies have shown that patients with schizophrenia fail to show this task-related increase in prefrontal functioning.

The study of schizophrenic patients at rest using blood flow techniques has suffered from criticism that rest is a complex mental state in its own right (18). Thus most studies have used some cognitive-activation paradigms that examine brain function at a fixed level of difficulty, usually at maximum accuracy in given cognitive tasks. Patients with schizophrenia almost invariably perform worse than healthy volunteers in almost any cognitive paradigm that has been imaged. Impaired performance of executive-function tasks has been associated with decreased activity in the dorsolateral prefrontal cortex (DLPFC) since the first groundbreaking studies in this area using the Wisconsin Card Sorting Test (19). The first fMRI report by YurgelunTodd et al. (20), showed left prefrontal underactivation during word generation. Although limited by the use of a surface coil, this study has been replicated (21), and reduced PFC activation during the Continuous Performance Test has also been demonstrated (22).

Later studies have related decreased DLPFC activation to impaired working memory performance (23). Callicott and colleagues, (24) using the N-back task, have reported decreased PFC activation in schizophrenic patients with impoverished working memory (24), but increased activation in those patients with relatively intact working memory (25). In the latter report, other areas evincing an abnormal response included the anterior cingulate cortex and the parietal cortex. The schizophrenic patients were treated with older-generation antipsychotics. The investigators increased the working memory load parametrically across multiple levels of load $(N)$ and contrasted the regions that increased as $N$ increased across groups. The authors confirmed the finding of reduced DLPFC activation and demonstrated that this result was specific to DLPFC in schizophrenics; that is, activations in Broca's area and in parietal, motor, and visual areas were normal. Decreased DLPFC activation correlated with both impaired working memory performance and ratings of behavioral disorganization in the schizophrenic patient group.

Using the Sternberg Item Recognition Paradigm, Manoach et al. $(26,27)$ have twice reported increased PFC activation in the face of diminished recall accuracy in schizophrenic patients. In the latter study, schizophrenic patients were found to activate the basal ganglia and thalamus, in contrast to control subjects. In addition to reduced DLPFC activity during word generation (noted above), Curtis et al. (28) found no difference in PFC activity during a semantic-decision task. However, reduced ventral PFC activation during a verbal working memory task has been demonstrated (29). On balance, these diverse findings argue in favor of PFC dysfunction as a characteristic of schizophrenia, but the particular mode (over- or underactivation) depends heavily on the nature and demands of the given task.

A study investigating the association between behavioral and neurophysiological measures of working memory task performance in 20 right-handed healthy male volunteers reported that prolonged reaction time to a verbal working memory task predicts increased power of posterior parietal cortical activation (30). This was consistent with prior studies that identified 
similar areas of parietal cortex as the site of the phonological-storage function in verbal working memory. In the study by Honey et al. (30), a spatially distributed network was activated, including DLPFC, inferior frontal gyrus, lateral premotor cortex, and supplementary motor area in the frontal lobes. More posteriorly, there were major foci of activation in parietal and occipitoparietal cortex, precuneus, lingual and fusiform gyri of the ventral occipital lobe, inferior temporal gyrus, and cerebellum. The power of functional response was positively correlated with reaction time in bilateral posterior parietal cortex, indicating that subjects who found the task difficult and responded with a slower reaction time tended to activate these regions more powerfully.

In schizophrenia research, interpretation of studies on working memory has been confounded by the fact that people with schizophrenia have impaired working memory function when the cognitive load exceeds the limited response capacity. We investigated working memory performance on a verbal N-back working memory task in 20 right-handed male subjects with chronic schizophrenia compared with 20 right-handed, age-matched, healthy male controls without personal or familial psychiatric history (30). Patients were selected on the basis of intact performance on a relatively low-load verbal working memory task, in order to take performance confounds into account. While there were no significant between-group differences in target identification, the schizophrenic patient group showed significantly increased mean response latency. Both groups demonstrated robust fronto-parietal activation. In the control subjects, the power of functional response was positively correlated with reaction time in bilateral posterior parietal cortex. However, this relationship of behavioral performance and cerebral response was not evident in the schizophrenic patients. It is possible that schizophrenic patients exhibit hypofrontality only when the physiological capacity of the PFC to respond to taskrelated requirements is exceeded by the cognitive load of working memory tasks.

\section{Social cognition}

Social cognition is what makes us human. To quote Steven Pinker in How the Mind Works (31), "We mortals cannot read other people's minds directly. But we make good guesses from what they say, what we read between the lines, what they show in their faces and eyes, and what best explains their behavior. It is our species' most remarkable talent." Social cognition refers to the ability to perceive, interpret, and respond appropriately to affective and other interpersonal cues. One of the least studied aspects of schizophrenia is emotional functioning, despite the fact that deficits in emotional experience, expression, and perception have been described for up to 100 years. Recently, however, the role of affect and affective-information processing in functional outcome has received greater attention. A deficit in the ability to process information related to affect may be a major factor in functional outcome. In addition to influencing social domains, affective-processing deficits may have wide-ranging impacts on functional outcome. Furthermore, social problem solving, a critical aspect of the maintenance of social and occupational relationships, is also substantially impaired in patients with schizophrenia. It is likely that lower-level aspects of social cognition, such as affect perception and expression, also impact social cognition. For instance, affect perception may be crucial to successful interactions, because the ability to perceive the listener's emotional reaction may be critical for the successful modulation of social and occupational interactions. It is undisputed that patients with schizophrenia have significant problems with interpersonal interactions $(32,33)$. These can manifest as poor premorbid social functioning, poor social functioning as the result of an exacerbation of symptoms, or a deficit state during remitted periods (e.g., anhedonia, the lack of ability to experience pleasure). Impairments in social functioning are indeed considered hallmarks of the disease.

Our group investigated social cognition in, to the best of our knowledge, the only study to date that has examined mental state attribution (MSA) in patients with schizophrenia (34). Using fMRI to explore abnormalities at the neural level in MSA, five individuals with schizophrenia were compared with seven matched controls. The neurocognitive network for normal MSA comprised middle, inferior frontal, and middle temporal regions. The normative network found for MSA was in line with previous findings (35). In the context of poor performance on this task, it was shown that patients with schizophrenia had significant reductions in neural activity in the left hemisphere and in the region of the middle and superior frontal lobes, bordering the insula (Figure 1). Reductions in the blood oxygen level-dependent (BOLD) response were specifically localized to the left inferior frontal gyrus (corresponding to Brodmann areas 44 and 45) relative to control subjects. The frontal underactivation in schizophrenia echoes functional and structural findings indicative of a frontal dysfunction in this patient population (36). This was the first demonstration of hypofrontality during social processing in schizophrenia (34).

These findings are consistent with Deakin's hypothesis (37) that dysfunction of a basolateral circuit involving ventral frontal and anterior temporal regions leads to disturbances of social communication and interpretation. A dysfunction of the frontal part of this network in schizophrenia appears to be sufficient to cause the social-interpretation deficits observed during the MSA task discussed above (34). A reduction in a strikingly similar left inferior frontal activation has also been shown in patients with autism, a condition characterized by theory-of-mind deficits. The integrity of left PFC therefore seems to be crucial for intact theoryof-mind processing, as has been previously suggested 
$(38,39)$. Left frontal underactivation in schizophrenia during MSA thus confirms the hypothesis of a socioemotional neurocognitive deficit in this patient group (34). Unlike in the study by Baron-Cohen et al. (35), there was no difference between the two groups in the region of the amygdala.

\section{The neural correlates of symptoms}

In a landmark paper, Liddle and colleagues (40) investigated the links between patterns of regional cerebral blood flow and schizophrenic symptoms. They identified three primary symptom clusters - psychomotor poverty, disorganization, and reality distortion and examined whether these symptoms were characterized by different patterns of cerebral perfusion. The results showed that reality-distortion scores were positively correlated with blood flow in the hippocampal region and the left PFC. Patient scores on the disorganization factor were associated with reduced blood flow in the right PFC, together with reduced activity of a left temporal lobe region involved in speech production. Lastly, psychomotor poverty was characterized by reduced activity in the left DLPFC and anterior cingulate. This finding provided support for the idea that negative symptoms are characterized by frontal lobe dysfunction.

Recent research has examined whether the major symptoms of schizophrenia can be linked to abnormal functioning of one or more brain regions. Answering this question may help to identify where antipsychotic drugs should be targeted. Perceptions of speech in the absence of an auditory stimulus (auditory verbal hallucinations) are a cardinal feature of schizophrenia. The initial studies that investigated the impact of positive symptoms, specifically auditory hallucinations, on cortical function used fMRI to replicate work done using radioactive imaging methods like positron emission tomography (PET). An early study demonstrated activations in subcortical nuclei (thalamic and striatal), limbic structures (especially the hippocampus), and paralimbic regions (parahippocampal and cingulate gyri, as well as orbitofrontal cortex) (41). David et al. (42) and Woodruff et al. (43) have found abnormal temporal cortex activation in response to external speech in patients with schizophrenia. In two schizophrenic patients with auditory hallucinations, David and colleagues found reduced activation in auditory cortex in response to auditory stimuli but no abnormalities in visual cortex in response to visual stimuli (42). Woodruff and colleagues replicated this reduction of auditory cortex response in a larger sample of patients with auditory hallucinations (43) and suggested that auditory hallucinations "competed" with external auditory stimuli for the cortical physiological response. In perhaps the most intriguing attempt to date to localize auditory hallucinations, Dierks et al. (44) studied three hallucinating patients using a modified event-related design. Patients indicated when they were hallucinating, and fMRI data from these temporal epochs were analyzed. The authors identified an area in the primary auditory cortex (Heschl's gyrus) that was also activated by external auditory stimuli, suggesting that such hallucinations involve primary auditory cortex dysfunction.

In 2000, Shergill and colleagues described a novel fMRI method permitting the measurement of spontaneous neural activity without the need for subjects to signal when hallucinations occurred (45). In six patients with schizophrenia experiencing frequent auditory hallucinations, the authors found that these hallucinations were associated with activation in the inferior frontal/insular, anterior cingulate, and temporal cortex bilaterally (with greater responses on the right), in the right thalamus and inferior colliculus, and in the left hippocampus and parahippocampal cortex. These findings suggest that a distributed network of cortical and subcortical areas may mediate auditory hallucinations. The notion that auditory hallucinations might be "inner speech" (thinking in words) was very recently taken further by this same group (46). Previous studies have shown that individuals with schizophrenia who were prone to auditory hallucinations demonstrated attenuated activation of brain areas during the monitoring of inner speech. Shergill et al. selected eight individuals with schizophrenia and a history of prominent auditory hallucinations and eight control participants and examined the rate of inner-speech generation, which was varied experimentally. The authors found that when the rate of inner-speech generation was increased, schizophrenic patients showed a relatively attenuated response in the right temporal, parietal, parahippocampal, and cerebellar cortex. The research to date supports the notion of abnormal activity of language- and speech-related areas of the brain in individuals with schizophrenia. It is possible that symptoms such as auditory hallucinations are due to a failure of self-monitoring such that inner speech is not recognized as such but instead is perceived as alien.

\section{A disorder of functional connectivity?}

Functional imaging research has moved away from looking at schizophrenia as a result of abnormalities in one or two regions - a lesion model - to looking at

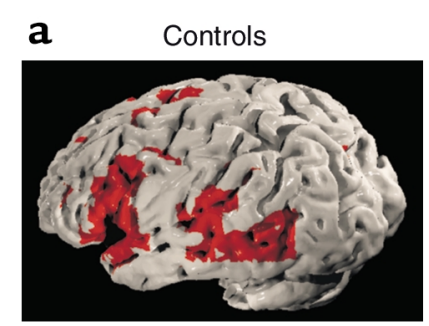

b Schizophrenic patients

\section{Figure 1}

Generic brain-activation maps for (a) control group and (b) patients with schizophrenia. View of the left hemisphere. Voxel-wise probability of type 1 error $=0.0004$. Reproduced with permission from The American Journal of Psychiatry (34). 
a

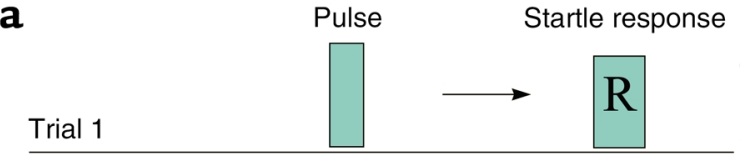

b

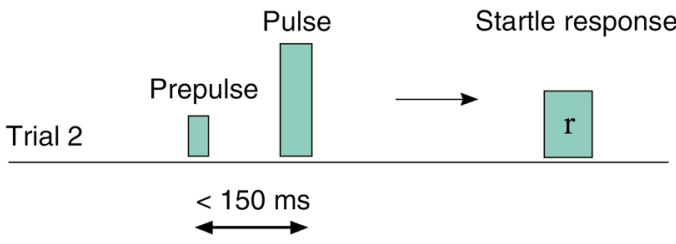

\section{Figure 2}

Diagrammatic representation of PPI. (a) Presentation of a strong stimulus, generally auditory or tactile, induces the startle response (R). (b) Exposure of the individual to a weaker prepulse less than $150 \mathrm{~ms}$ before exposure to the stronger pulse results in a reduced startle response $(r)$.

changes in neural circuits (47). Most brain regions are highly interconnected, and most tasks recruit the involvement of spatially distributed structures. Functional disconnectivity implies that the normal pattern of distributed activation, and the relationship(s) between activation in regions, are abnormal. Functional disconnectivity may provide an explanation for many of the symptoms and cognitive deficits associated with schizophrenia (48). Although there is relatively widespread agreement that functional connectivity is abnormal in schizophrenia, debate continues about which circuits are abnormal. Subcortical limbic structures (e.g., the amygdala) connect to the orbitofrontal cortex, and information is transferred to the medial frontal lobe to make social cognition decisions. Neuroimaging studies of social cognition also show us that it is necessary for the subcortical and frontal systems to work in tandem (34).

\section{Translational medicine and drug discovery}

The ability to translate animal models of cognitive deficits to humans and ultimately to schizophrenic patients is likely to allow us to discover the neural correlates of these deficits. Prepulse inhibition (PPI) of the startle reflex is one such mechanism. This is the ability of a weak stimulus, the prepulse, to reduce the startle response to a strong startling stimulus, the pulse, that follows closely (by $30-500 \mathrm{~ms}$ ) (49) (Figure 2). Presentation of the pulse at short lead intervals, while the prepulse is still being analyzed, causes impaired processing of, and thus an attenuated overt response to, this stimulus. This phenomenon is thought to reflect a sensorimotor gating mechanism and to serve the function of avoiding behavioral interference that might otherwise result from the simultaneous processing of discrete stimuli. Deficits in the ability to avoid such stimulus interference are thought to lead to sensory overstimulation and behavioral confusion (50), as seen for example in schizophrenia $(51,52)$.
PPI is observed in both human and animal subjects (53), including invertebrates (54). There is evidence from animal studies that PPI is mediated by brain stem circuits involving the inferior colliculus (mainly for acoustic stimuli), superior colliculus, pedunculopontine tegmental nucleus, laterodorsal tegmental nucleus, substantia nigra pars reticulata, and caudal pontine reticular nucleus (55), and modulated by forebrain circuits involving the PFC, thalamus, hippocampus, amygdala, nucleus accumbens, striatum, ventral pallidum, globus pallidus, and subpallidal efferents to the pedunculopontine nucleus (56-58). Consistent with the known neural substrates of PPI in the rat, deficient PPI is observed in a number of psychiatric and neurological disorders characterized by abnormalities at some level in the cortico-striato-thalamo-pallido-pontine circuitry, including schizophrenia (59-65), Huntington disease (66), obsessive-compulsive disorder (OCD) (67), attention-deficit hyperactivity disorder (68), and Tourette syndrome (69).

Whole-brain fMRI has been employed to elucidate the neural correlates of PPI using air-puff stimuli as both the prepulse and the pulse in groups of healthy subjects and schizophrenic patients (70). In the past, the paradigms most commonly used to demonstrate PPI of the startle response used a strong noise burst as the pulse and a weak noise burst as the prepulse. PPI using an acoustic prepulse combined with an air puff to elicit startle has also been reported to be deficient in patients with schizophrenia $(50,71)$. We developed a tactile version of the PPI paradigm (air puffs to the sternum) suitable for AMRI investigations (70). Using the tactile PPI paradigm, we applied fMRI to examine and compare the neural correlates in normal healthy subjects with those in patients with schizophrenia. Healthy subjects showed, in association with PPI, increased BOLD response bilaterally in the striatum (extending to hippocampus and thalamus), the right inferior frontal gyrus, bilateral inferior parietal lobe, and supramarginal gyrus (70). Relative to the control group, a significantly reduced BOLD response in all such areas was reported in patients with schizophrenia, who also showed considerably (though not significantly) lower PPI. In general, these findings were consistent with the neural substrates that regulate PPI in the rat (58), with previous studies that investigated neural correlates of PPI in normal and schizophrenic subjects using PET (72), and with more recent studies that explored neural correlates of PPI in normal subjects using fMRI (73). However, our study (70) is the first, to our knowledge, to reveal evidence for the involvement of basal ganglia structures and the hippocampus in human PPI. These brain regions not only are known to modulate PPI in the rat (58) but have also been implicated in the pathophysiology and treatment of schizophrenia (74-80). Previous neuroimaging investigations in human subjects used a region-of-interest approach that did not include the areas reported here. 
a

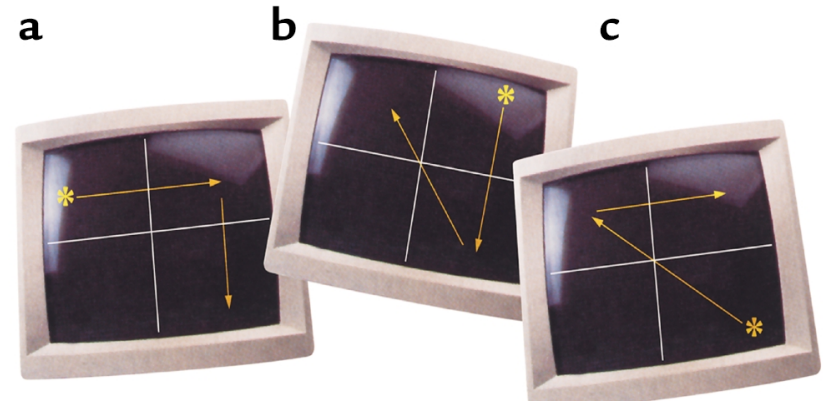

Figure 3

An illustration of pattern-trial rules used in a PL test. The asterisklike target moves among four locations on the screen, which is divided into four equal quadrants by two intersecting white lines. The movement of the target during the pattern-trial test is predictable in $75 \%$ of cases, as determined by three specific rules: (a) a horizontal target movement is followed by a vertical target movement, (b) a vertical target movement is followed by a diagonal target movement, and (c) a diagonal target movement is followed by a horizontal movement. The fourth movement of the target during the pattern trials is unpredictable. In the control state, individuals are subjected to only random target movements. During the experimental test, individuals are subjected to blocks of patterns and required to determine the expected projectory of imminent movement, and their subsequent reaction time is recorded. Reproduced with permission from Schizophrenia Research (84).

It is important to note that the patients included in this study (70) were on older, or conventional, antipsychotic medication. The differences between the schizophrenic patients and controls in activity of the striatal, thalamic, hippocampal, and frontal regions most likely reflect an aspect of the schizophrenic illness process, reflected as relatively low PPI. However, it remains possible that these differences are direct effects of conventional antipsychotic medication. Further fMRI studies of PPI are now warranted in (a) healthy subjects treated with conventional and atypical antipsychotics; (b) drug-naive patients with schizophrenia; (c) patients with schizophrenia receiving conventional and atypical antipsychotic drugs; and (d) nonschizophrenic psychiatric patients exhibiting deficient PPI, such as individuals suffering from OCD. In all cases, increased sampling of both sexes will be required to extend and refine these findings.

Another clinical predictor of functional outcome in schizophrenia is the ability to learn. Procedural learning (PL) is a form of skill acquisition in which learning occurs as a function of practice of the task, without the need for conscious awareness of the learned skill or routine $(81,82)$. In normal subjects, PL is found to be independent of intelligence and also of performance on tests of declarative learning and memory, such as recall or recognition, in which performance depends on the knowledge of facts and often correlates with intelligence (83). The functional neuroanatomy of PL has not been fully established using fMRI. Schizophrenic patients often (though not invariably) have been found to show impaired PL.
Kumari et al. (84) performed fMRI on healthy individuals, and schizophrenic patients on conventional antipsychotics, during a blocked, periodic sequencelearning task (Figure 3) and revealed that while healthy subjects demonstrated significant PL (Figure 4a), schizophrenic patients did not (Figure 4b). In healthy subjects, PL was associated with increased activation in the striatum, thalamus, cerebellum, precuneus, medial frontal lobe, and cingulate gyrus. The power of activation in the thalamus, striatum, precuneus, cingulate gyrus, and Brodmann area 6 was related to the magnitude of PL in these individuals. No regions, except the anterior inferior gyrus, were significantly activated in schizophrenic patients. The degree of activation of the caudate nucleus, thalamus, precuneus, and sensorimotor regions was significantly different between the two groups. The data demonstrate the involvement of the striatum, cerebellum, thalamus, cingulate gyrus, precuneus, and sensorimotor regions in PL. Schizophrenic patients had diminished PL scores and showed a lack of activation in relevant brain regions. The involvement of frontal regions in PL may occur, as suggested earlier, via their connections to the basal ganglia. Alternatively, these regions may be directly involved in attention and error-checking mechanisms required in rapid learning (85). The study by Kumari et al. (84), however, involved a low number of schizophrenic patients with mild symptoms and was limited to male subjects. Although the study helps to identify the brain regions involved in PL, it does not provide conclusive evidence that impaired PL in schizophrenia results from conventional antipsychotics or reflects an aspect of the disease process. Further fMRI studies of PL in normal subjects treated with conventional antipsychotics, a

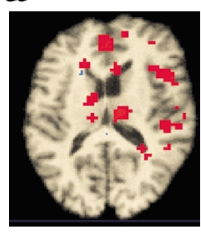

$20 \mathrm{~mm}$ b

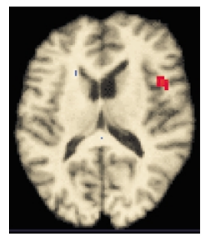

$20 \mathrm{~mm}$

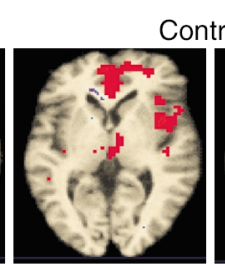

$9 \mathrm{~mm}$

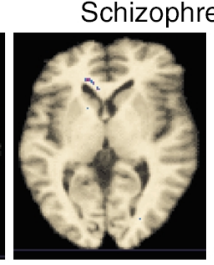

$9 \mathrm{~mm}$
Controls

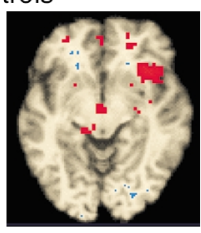

$-1 \mathrm{~mm}$

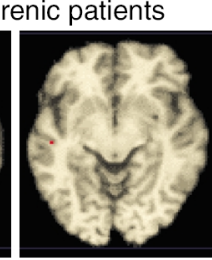

$-1 \mathrm{~mm}$

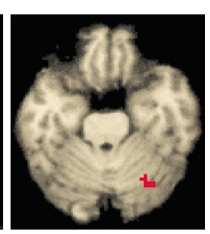

$-18 \mathrm{~mm}$

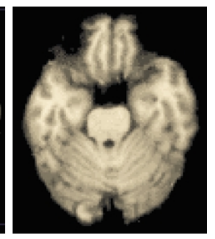

$-18 \mathrm{~mm}$

\section{Figure 4}

Generic brain-activation maps in response to a PL task. (a) In healthy subjects, regions of activation are demonstrated in the striatum, thalamus, cingulate gyrus, insula, and cerebellum. (b) In patients with schizophrenia, there is a lack of activation in these same regions. Below each image, $z$ coordinates are shown, left to right. Reproduced with permission from Schizophrenia Research (84). 
$\mathbf{a}$

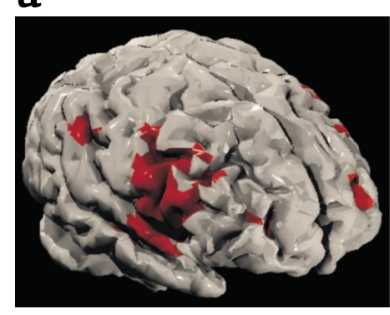

b

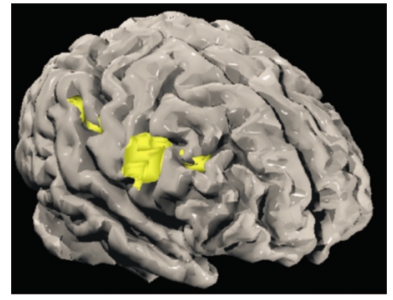

Figure 5

Rendered images of the brain showing regions of increased activity following substitution of risperidone for typical antipsychotic drugs. (a) Brain activity related to a memory task in schizophrenic patients. (b) Specific areas of improved brain function following treatment with risperidone. Reproduced with permission from Remedica Publishing Ltd. (90).

drug-naive patients, and schizophrenic patients given atypical antipsychotics would help to clarify the roles of schizophrenic disease processes and antipsychotic medication in impaired PL and associated brain abnormalities in schizophrenia.

\section{Functional MRI of drug effects}

There is a large body of evidence regarding the different pharmacological profiles of the older "typical" and the newer "atypical" antipsychotic drugs; however, relatively little is known about their differential effects on cerebral function. The ability to monitor the effects of antipsychotic drugs on brain activity is likely to greatly aid our understanding of how drugs work, and to help us to identify which drug would be most suitable for a particular patient. Researchers are beginning to use fMRI to examine the effects of different antipsychotic drugs on cerebral activity.

A recent study by our group used fMRI to study whether switching schizophrenics from typical antipsychotic drugs to the atypical drug risperidone would produce differences in brain activity during a working memory task (86). Working memory deficits have been consistently reported in schizophrenia, and there is evidence of functional hypofrontality during working memory tasks (87). Working memory performance has been shown to be related to prefrontal dopamine function (88), and given that there is evidence from animal studies that atypical antipsychotics increase prefrontal dopamine transmission (89), the authors predicted that switching schizophrenics from typical antipsychotics to risperidone would result in the increased activity of prefrontal regions. Patients were scanned at a base-line assessment when all were on typical antipsychotic drugs. Subsequently, half of the schizophrenic group was switched to risperidone while the other half remained on their typical medication. Subjects were rescanned after 6 weeks. Patients switched to risperidone exhibited an increase in blood oxygenation in the right DLPFC, precuneus, and supplementary motor area at follow-up (86, 90) (Figure 5). The cause of the observed increase in frontal cortical activity may reflect the effect of dopamine 2 receptor antagonism, a reduction in the activity of inhibitory $\gamma$-aminobutyric acid neurons via serotonergic modulation, or, alternatively, a serotoninmediated increase in frontal dopamine activity.

This shows that it may be possible to reactivate brain regions in psychiatric illness via drug treatment. The increased activation in prefrontal regions may help to explain improvements in cognitive functioning that have been observed after treatment with risperidone (91). The study discussed above (86) also demonstrates the suitability of fMRI as a tool to assess psychopharmacological effects. Given that fMRI has many advantages over radioactive techniques for longitudinal study, this approach could be used to study drug effects in other psychiatric disorders, such as the effects of cholinesterase inhibitors in Alzheimer disease.

\section{Conclusions}

In the field of functional brain imaging, much interest is currently being focused on fMRI as a tool that will greatly enhance our understanding of normal brain functioning and how it may differ in psychiatric illnesses. PMRI might be sensitive to the functional consequences of these disorders, where neuropsychological testing alone might miss such pathophysiology. A growing area of research involves the linking of changes in brain function - in either receptor functioning or blood flow - with medication status, clinical improvement, and cognitive functioning. Understanding the link between medication and brain function is vital to the development of the next generation of antipsychotic drugs. Given the large number of brain structures that have been reported to be abnormal in schizophrenia, the focus of research has switched from lesion models of psychosis to theories of disordered neural circuitry. There is already evidence from both structural and functional studies of abnormal connections and interactions between brain regions in schizophrenia, which may help to explain the symptoms and cognitive deficits associated with the disease.

Imaging has transformed our understanding of the mechanisms of action of antipsychotic medications, and therefore their clinical use. However, many questions regarding clinical practice remain to be answered. Why does a patient who has remained stable on medication for months, or possibly years, begins to develop symptoms again? What can predict relapse, and how can we best treat it? Why do some patients respond to one type of medication but not to another? Why are some patients unresponsive to any currently available antipsychotics? At the moment, we have very little understanding of the neurobiological correlates of relapse, remission, and treatment response in schizophrenia. Neuroimaging offers us the possibility of answering these questions, and therefore of improving the quality of patient care.

Neuronal plasticity is now a recognized feature of the adult brain. An important aspect of future investigations might be the possible remediation of cognition and the 
reawakening of abnormal areas in schizophrenia with new-generation antipsychotics, cognitive enhancers, and psychological cognitive-remediation techniques.

\section{Acknowledgments}

Part of this work was made possible by funding from the National Alliance for Research on Schizophrenia and Depression, USA, and PsychMed Ltd. The author is grateful to Veena Kumari for the variety of intellectual input to his group's research.

1. Rund, B.R., and Borg, N.E. 1999. Cognitive deficits and cognitive training in schizophrenic patients: a review. Acta Psychiatr. Scand. 100:85-95.

2. Hegarty, J.D., et al. 1994. One hundred years of schizophrenia: a metaanalysis of the outcome literature. Am. J. Psychiatry. 151:1409-1416.

3. Crow, T.J., Done, D.J., and Sacker, A. 1995. Childhood precursors of psychosis as clues to its evolutionary origins. Eur. Arch. Psychiatry Clin. Neurosci. 245:61-69.

4. Friedman, J.I., et al. 2001. Six-year follow-up study of cognitive and functional status across the lifespan in schizophrenia: a comparison with Alzheimer's disease and normal aging. Am. J. Psychiatry. 158:1441-1448.

5. Palmer, B.W., et al. 1997. Is it possible to be schizophrenic yet neuropsychologically normal? Neuropsychology. 11:437-446.

6. Harvey, P.D., et al. 1996. Cognitive impairment and negative symptoms in geriatric chronic schizophrenic patients: a follow-up study. Schizophr. Res. 22:223-231.

7. Hughes, C., et al. 2003. Longitudinal study of symptoms and cognitive function in chronic schizophrenia. Schizophr. Res. 59:137-146.

8. Green, M.F. 1996. What are the functional consequences of neurocognitive deficits in schizophrenia? Am. J. Psychiatry. 153:321-330.

9. Kraepelin, E. 1919. Dementia praecox and paraphrenia. Robert E. Krieger Publishing Co. Huntington, New York, USA.

10. Selemon, L.D., Rajkowska, G., and Goldman-Rakic, P.S. 1995. Abnormally high neuronal density in the schizophrenic cortex: a morphometric analysis of prefrontal area 9 and occipital area 17. Arch. Gen. Psychiatry. 52:805-818.

11. Selemon, L.D., Rajkowska, G., and Goldman-Rakic, P.S. 1998. Elevated neuronal density in prefrontal area 46 in brains from schizophrenic patients: application of a three-dimensional, stereologic counting method. J. Comp. Neurol. 392:402-412.

12. Woo, T.U., et al. 1998. A subclass of prefrontal gamma-aminobutyric acid axon terminals are selectively altered in schizophrenia. Proc. Natl. Acad. Sci. U. S. A. 95:5341-5346.

13. Akbarian, S., et al. 1996. Selective alterations in gene expression for NMDA receptor subunits in prefrontal cortex of schizophrenics. J. Neurosci. 16:19-30.

14. Benes, F.M. 1991. Deficits in small interneurons in prefrontal and cingulate cortices of schizophrenic and schizoaffective patients. Arch. Gen. Psychiatry. 48:996-1001.

15. Ingvar, D.H., and Franzen, G. 1974. Abnormalities of cerebral blood flow distribution in patients with chronic schizophrenia. Acta Psychiatr. Scand. 50:425-462.

16. Taylor, S., Tandon, R., and Koeppe, R. 1997. PET study of greater visual activation in schizophrenia. Am. J. Psychiatry. 154:1296-1298.

17. Gur, R.C., and Gur, R.E. 1995. Hypofrontality in schizophrenia: RIP. Lancet. 345:1383-1340.

18. Weinberger, D.R., and Berman, K.F. 1996. Prefrontal function in schizophrenia: confounds and controversies. Philos. Trans. R. Soc. Lond. B Biol. Sci. 351:1495-1503.

19. Weinberger, D.R., Berman, K.F., and Zec, R.F. 1986. Physiologic dysfunction of dorsolateral prefrontal cortex in schizophrenia. I. Regional cerebral blood flow evidence. Arch. Gen. Psychiatry. 43:114-124.

20. Yurgelun-Todd, D.A., et al. 1996. Functional magnetic resonance imaging of schizophrenic patients and comparison subjects during word production. Am. J. Psychiatry. 153:200-205.

21. Curtis, V.A., et al. 1998. Attenuated frontal activation during a verbal fluency task in patients with schizophrenia. Am. J. Psychiatry. 155:1056-1063.

22. Volz, H., et al. 1999. Decreased frontal activation in schizophrenics during stimulation with the Continuous Performance Test: a functional magnetic resonance imaging study. Eur. Psychiatry. 14:17-24.

23. Barch, D., et al. 2001. Selective deficits in prefrontal cortex function in medication-naive patients with schizophrenia. Arch. Gen. Psychiatry. 58:280-288.

24. Callicott, J.H., et al. 1998. Functional magnetic resonance imaging brain mapping in psychiatry: methodological issues illustrated in a study of working memory in schizophrenia. Neuropsychopharmacology. 18:186-196
25. Callicott, J.H., et al. 2000. Physiological dysfunction of the dorsolateral prefrontal cortex in schizophrenia revisited. Cereb. Cortex. 10:1078-1092.

26. Manoach, D.S., et al. 1999. Schizophrenic subjects activate dorsolateral prefrontal cortex during a working memory task, as measured by fMRI. Biol. Psychiatry. 45:1128-1137.

27. Manoach, D.S., et al. 2000. Schizophrenic subjects show aberrant fMRI activation of dorsolateral prefrontal cortex and basal ganglia during working memory performance. Biol. Psychiatry. 48:99-109.

28. Curtis, V.A., et al. 1999. Attenuated frontal activation in schizophrenia may be task dependent. Schizophr. Res. 37:35-44.

29. Stevens, A.A., et al. 1998. Cortical dysfunction in schizophrenia during auditory word and tone working memory demonstrated by functional magnetic resonance imaging. Arch. Gen. Psychiatry. 55:1097-1103.

30. Honey, G., Bullmore, E., and Sharma, T. 2001. De-coupling of cognitive performance and cerebral functional response during working memory in schizophrenia. Schizophr. Res. 53:45-56.

31. Pinker, S. 1999. How the mind works. W.W. Norton and Co. New York, New York, USA. 660 pp.

32. Green, M.F., et al. 2000. Neurocognitive deficits and functional outcome in schizophrenia: are we measuring the "right stuff"? Schizophr. Bull. 26:119-136.

33. Mueser, K.T. 2000. Cognitive functioning, social adjustment and longterm outcome in schizophrenia. In Cognition in schizophrenia. T. Sharma and P. Harvey, editors. Oxford University Press. Oxford, United Kingdom. 157-177.

34. Russell, T.A., et al. 2000. Exploring the social brain in schizophrenia: left prefrontal underactivation during mental state attribution. Am. J. Psychiatry. 157:2040-2042.

35. Baron-Cohen, S., et al. 1999. Social intelligence in the normal and autistic brain: an fMRI study. Eur. J. Neurosci. 11:1891-1898.

36. Harrison, P. 1999. The neuropathology of schizophrenia: a critical review of the data and their interpretation. Brain. 122:593-624.

37. Deakin, J.F.W. 1994. Neuropsychological implications of brain changes in schizophrenia. Psychopathology. 27:251-254.

38. Frith, C.D. 1996. Brain mechanisms for having a "theory of mind." J. Psychopharmacol. 19:9-15.

39. Goel, V., Grafman, J., Sadato, N., and Hallett, M. 1995. Modeling other minds. Neuroreport. 6:1741-1746.

40. Liddle, P.F., et al. 1992. Patterns of cerebral blood flow in scizophrenia. Br. J. Psychiatry. 160:179-186.

41. Silbersweig, D.A., et al. 1995. A functional neuroanatomy of hallucinations in schizophrenia. Nature. 378:176-179.

42. David, A.S., et al. 1996. Auditory hallucinations inhibit exogenous activation of auditory association cortex. Neuroreport. 7:932-936.

43. Woodruff, P.W.R., et al. 1997. Auditory hallucinations and the temporal cortical response to speech in schizophrenia: a functional magnetic resonance imaging study. Am. J. Psychiatry. 154:1676-1682.

44. Dierks, T., et al. 1999. Activation of Heschl's gyrus during auditory hallucinations. Neuron. 22:615-621.

45. Shergill, S.S., Brammer, M.J., Williams, S.C., Murray, R.M., and McGuire, P.K. 2000. Mapping auditory hallucinations in schizophrenia using functional magnetic resonance imaging. Arch. Gen. Psychiatry. 57:1033-1038.

46. Shergill, S.S., et al. 2003. Engagement of brain areas implicated in processing inner speech in people with auditory hallucinations. Br. J. Psychiatry. 182:525-531.

47. Sharma, T., and Sheringham, J. 2002. Brain imaging in psychiatry: what has it done for the patient? Hosp. Med. 63:326-327.

48. Bullmore, E., et al. 2000. How good is good enough in path analysis of fMRI data? Neuroimage. 11:289-301.

49. Graham, F.K. 1975. The more or less startling effects of weak prestimuli. Psychophysiology. 12:238-248.

50. Braff, D.L., and Geyer, M.A. 1990. Sensorimotor gating and schizophrenia: human and animal model studies. Arch. Gen. Psychiatry. 47:181-188.

51. Perry, W., Geyer, M.A., and Braff, D.L. 1999. Sensorimotor gating and thought disturbance measured in close temporal proximity in schizophrenic patients. Arch. Gen. Psychiatry. 56:277-281.

52. Dawson, M.E., Schell, A.M., Hazlett, E.A., Nuechterlein, K.H., and Filion, D.L. 2000. On the clinical and cognitive meaning of impaired sensorimotor gating in schizophrenia. Psychiatry Res. 96:187-197.

53. Hoffman, H.S., and Ison, J.R. 1992. Reflex modification and the analysis of sensory processing in developmental and comparative research. In Attention and information processing in infants and adults: perspectives from human and animal research. B.A. Campbell, H. Hayne, and R. Richardson, editors. Lawrence Erlbaum Associates Inc. Hillsdale, New Jersey, USA. 83-111.

54. Mongeluzi, D.L., Hoppe, T.A., and Frost, W.N. 1998. Prepulse inhibition of the Tritonia escape swim. J. Neurosci. 15:8467-8472.

55. Fendt, M., Liang, L., and Yeomans, J.S. 2001. Brain stem circuits mediating prepulse inhibition of the startle reflex. Psychopharmacology. 156:216-224.

56. Koch, M., and Schnitzler, H. 1997. The acoustic startle response in rats: 
circuits mediating evocation, inhibition and potentiation. Behav. Brain Res. 89:35-49.

57. Swerdlow, N.R., and Geyer, M.A. 1998. Using an animal model of deficient sensorimotor gating to study the pathophysiology and new treatments of schizophrenia. Schizophr. Bull. 24:285-301.

58. Swerdlow, N.R., Geyer, M.A., and Braff, D.L. 2001. Neural circuit regulation of prepulse inhibition of startle in the rat: current knowledge and future challenges. Psychopharmacology. 156:194-215.

59. Braff, D.L., et al. 1978. Prestimulus effects in human startle reflex in normals and schizophrenics. Psychophysiology. 15:339-343.

60. Braff, D.L., Grillon, C., and Geyer, M.A. 1992. Gating and habituation of the startle reflex in schizophrenic patients. Arch. Gen. Psychiatry. 49:206-215.

61. Braff, D.L., Swerdlow, N.R., and Geyer, M.A. 1999. Symptom correlates of prepulse inhibition deficits in male schizophrenic patients. Am. J. Psychiatry. 156:596-602.

62. Grillon, C., et al. 1992. Startle gating deficit occurs across prepulse intensities in schizophrenic patients. Biol. Psychiatry. 32:929-943.

63. Kumari, V., Soni, W., and Sharma, T. 1999. Normalization of information processing deficits in schizophrenia by clozapine. Am. J. Psychiatry. 156:1046-1051.

64. Kumari, V., Soni, W., Mathew, V.M., and Sharma, T. 2000. Prepulse inhibition of the startle response in men with schizophrenia: effects of age of onset of illness, symptoms, and medication. Arch. Gen. Psychiatry. 57:609-614.

65. Kumari, V., Soni, W., and Sharma, T. 2002. Prepulse inhibition of the startle response in risperidone-treated patients: comparison with typical antipsychotics. Schizophr. Res. 55:139-146.

66. Swerdlow, N.R., Filion, D., Geyer, M.A., and Braff, D.L. 1995. Normal personality correlates of sensorimotor, cognitive, and visuospatial gating. Biol. Psychiatry. 37:286-299.

67. Swerdlow, N.R., et al. 1993. A preliminary assessment of sensorimotor gating in patients with obsessive compulsive disorder. Biol. Psychiatry. 33:298-301.

68. Ornitz, E.M., Hanna, G.L., and de Traversay, J. 1992. Prestimulationinduced startle modulation in attention-deficit hyperactivity disorder and nocturnal enuresis. Psychophysiology. 29:437-451.

69. Castellanos, F.X., et al. 1996. Sensorimotor gating in boys with Tourette's syndrome and ADHD. Biol. Psychiatry. 39:33-41.

70. Kumari, V., et al. 2003. Neural correlates of tactile prepulse inhibition: a functional MRI study in normal and schizophrenic subjects. Psychiatry Res. 122:99-113.

71. Perry, W., and Braff, D. 1994. Information-processing deficits and thought disorder in schizophrenia. Am. J. Psychiatry. 151:363-367.

72. Hazlett, E.A., et al. 1998. Prefrontal cortex glucose metabolism and startle eyeblink modification abnormalities in unmedicated schizophrenia patients. Psychophysiology. 35:186-198.

73. Hazlett, E.A., et al. 2001. Thalamic activation during an attention-to-prepulse startle modification paradigm: a functional MRI study. Biol. Psychiatry. 50:281-291.
74. Gray, J.A., Feldon, J., Rawlins, J.N.P., Hemsley, D.R., and Smith, A.D 1991. The neuropsychology of schizophrenia. Behav. Brain Sci. 14:1-84.

75. Pantelis, C., et al. 2003. Neuroanatomical abnormalities before and after onset of psychosis: a cross-sectional and longitudinal MRI comparison. Lancet. 361:281-288.

76. Lauer, M., Senitz, D., and Beckmann, H. 2001. Increased volume of the nucleus accumbens in schizophrenia. J. Neural Transm.. 108:645-660.

77. Scarr, E., Copolov, D.L., and Dean, B. 2001. A proposed pathological model in the hippocampus of subjects with schizophrenia. Clin. Exp. Pharmacol. Physiol. 28:70-73.

78. Shenton, M.E., Dickey, C.C., Frumin, M., and McCarley, R.W. 2001. A review of MRI findings in schizophrenia. Schizophr. Res. 49:1-52.

79. Shihabuddin, L., et al. 2001. Striatal size and relative glucose metabolic rate in schizotypal personality disorder and schizophrenia. Arch. Gen. Psychiatry. 58:877-884.

80. Xiberas, X., et al. 2001. Extrastriatal and striatal D(2) dopamine receptor blockade with haloperidol or new antipsychotic drugs in patients with schizophrenia. Br. J. Psychiatry. 179:503-508.

81. Cohen, N.J., and Squire, L.R. 1980. Preserved learning and knowledge of analyzing skills in amnesia: dissociation of knowing how and knowing what. Science. 201:207-209.

82. Squire, L.R., and Zola-Morgan, S. 1988. Memory: brain systems and behaviour. Trends Neurosci. 11:170-175.

83. Feldman, K.J., Kerr, B., and Streissguth, A.P. 1995. Correlational analysis of procedural and declarative learning performances. Intelligence. 20:87-114.

84. Kumari, V., et al. 2002. Procedural learning in schizophrenia: a functional magnetic resonance imaging investigation. Schizophr. Res. 57:97-107.

85. Grafton, S.T., Woods, R.P., and Tyszka, M. 1994. Functional imaging of procedural motor learning: relating cerebral flow with individual subject performance. Hum. Brain Mapp. 1:221-234.

86. Honey, G.D., et al. 1999. Differences in frontal cortical activation by a working memory task after substitution of risperidone for typical antipsychotic drugs in patients with schizophrenia. Proc. Natl. Acad. Sci. U. S. A. 96:13432-13437.

87. Stevens, A.A., Goldman-Rakic, P.S., Gore, J.C., Fulbright, R.K., and Wexler, B.E. 1998. Cortical dysfunction in schizophrenia during auditory word and tone working memory demonstrated by functional magnetic resonance imaging. Arch. Gen. Psychiatry. 55:1097-1103.

88. Watanabe, M., Kodama, T., and Hikosaka, K. 1997. Increase of extracellular dopamine in primate prefrontal cortex during a working memory task. J. Neurophysiol. 78:2795-2798.

89. Hertel, P., Nomikos, G.G., Iurlo, M., and Svensson, T.H. 1996. Risperidone: regional effects in vivo on release and metabolism of dopamine and serotonin in the rat brain. Psychopharmacology. 124:74-86.

90. Sharma, T., and Chitnis, X. 2001. Brain imaging in schizophrenia: insights and applications. Remedica Publishing Ltd. London, United Kingdom. p. 51.

91. Meltzer, H.Y., and McGurk, S.R. 1999. The effects of clozapine, risperidone, and olanzapine on cognitive function in schizophrenia. Schizophr. Bull. 25:233-255. 